\title{
Enfermeiro de Saúde da Família na Amazônia: conceitos e manejo na temática do uso de álcool
}

\author{
FAMILY HEALTH NURSE IN THE AMAZON: CONCEPTS AND MANAGEMENT OF THEMES \\ REGARDING ALCOHOL USE
}

\section{ENFERMERO DE SALUD DE LA FAMILIA EN AMAZONIA: CONCEPTOS Y MANEJO EN LA TEMÁTICA DEL ALCOHOLISMO}

\section{Rodrigo Otávio Moretti-Pires ${ }^{1}$, Sidnei Bruno Guedes Ferro², Fátima Büchele ${ }^{3}$, Hadelândia Milon de Oliveira $^{4}$, Maria Jacirema Ferreira Gonçalves ${ }^{5}$}

\section{RESUMO}

O estudo visa identificar a concepção dos enfermeiros sobre a temática do uso problemático de álcool e as formas de manejo utilizado, em um contexto amazônico. Utilizaram-se grupo focal e entrevistas individuais com todos os enfermeiros das doze equipes de saúde da família de um município do interior da Amazônia, Brasil. Observou-se falta de formação universitária, de educação permanente e de suporte/ referência - contra - referência na atenção à dimensão do uso de álcool na população adscrita. Há necessidade de reformulação da estrutura curricular dos cursos de graduação em enfermagem, assim como educação permanente para as Equipes de Saúde da Família e suporte nesta importante temática e suas conseqüências, tanto para o indivíduo e para as famílias, como para a sociedade em geral.

\section{DESCRITORES}

Alcoolismo

Saúde da família

Enfermagem em saúde pública

Educação em enfermagem

\begin{abstract}
The objective of this study is to identify the concept that nurses in the Amazon have on alcohol abuse and the forms used for management. Focal group and individual interviews were performed with all nurses of twelve family health teams from a city in the Amazon, Brazil. It was observed there was a lack of university degrees, continuing education and of support/reference counter - reference towards the dimension of alcohol use in the studied population. There is need to reform nursing courses and perform continuing education for family health team members, and provide them with the necessary support regarding this important theme and its consequences, for individuals and their families, as well as for the society as a whole.
\end{abstract}

\author{
DESCRIPTORS \\ Alcoholism \\ Family health \\ Public health nursing \\ Education, nursing
}

\begin{abstract}
RESUMEN
El estudio apunta a identificar la concepción de los enfermeros sobre la temática del abuso de alcohol y las formas de manejo utilizadas, en el contexto Amazónico. Se utilizó grupo focal y entrevistas individuales con todos los enfermeros de los doce equipos de salud familiar de un municipio del interior de Amazonia, Brasil. Se observó la falta de formación universitaria, de educación permanente y de soportereferencia-contrarreferencia en la atención de la dimensión del abuso de alcohol en la población descripta. Existe necesidad de reformulación de la estructura curricular de los cursos de graduación en enfermería, así como educación permanente en Equipos de Salud Familiar y soporte para los mismos en esta importante temática y sus consecuencias, tanto individuales como familiares y para la sociedad en general.
\end{abstract}

\section{DESCRIPTORES \\ Alcoholismo \\ Salud de la família \\ Enfermería en salud públic \\ Educación en enfermería}

\footnotetext{
${ }_{1}^{1}$ Professor Doutor do Departamento de Saúde Pública do Centro de Ciências da Saúde da Universidade Federal de Santa Catarina. Florianópolis, SC, Brasil. rodrigomoretti@ccs.ufsc.br ${ }^{2}$ Graduando do Instituto de Saúde e Biotecnologia da Universidade Federal do Amazonas. Manaus, AM, Brasil. brunoferr@gmail.com ${ }^{3}$ Professora Doutora do Departamento de Saúde Pública do Centro de Ciências da Saúde da Universidade Federal de Santa Catarina. Florianópolis, SC, Brasil. buchele@mbox1.ufsc.br ${ }^{4}$ Professora Assistente do Departamento de Enfermagem em Clínica Médica da Escola de Enfermagem de Manaus da Universidade Federal do Amazonas. Manaus, AM, Brasil. hmilon@ufam.edu.br ${ }^{5}$ Professora Doutra do Departamento de Enfermagem Materno Infantil e Saúde Pública da Escola de Enfermagem de Manaus da Universidade Federal do Amazonas. Manaus, AM, Brasil. jaciremagoncalves@ufam.edu.br
} 


\section{INTRODUÇÃO}

O Ministério da Saúde (MS) desenvolveu diversas estratégias para modificar as práticas tradicionais de saúde na Atenção Primária à Saúde (APS), no sentido de melhorar acolhimento, vínculo entre os usuários e equipes. Estas iniciativas esbarram na questão da formação do profissional no seio da universidade ${ }^{(1)}$. Mudanças nos cursos de graduação da área da saúde foram propostas, com vistas a um maior comprometimento do futuro profissional com os princípios do Sistema Único de Saúde (SUS) durante sua formação, contexto importante para o desenvolvimento da Estratégia Saúde da Família (ESF).

Apesar da implantação da Saúde da Família, com a finalidade de reorganizar os processos de trabalho e ações do SUS, a formação pr ofissional brasileira em saúde da atualidade, mesmo quando objetivando estratégias como a ESF, promove uma visão descontextualizada da realidade brasileira $^{(2-3)}$, existindo contradições entre as instituições de ensino superior, os serviços de saúde e as comunidades. Os profissionais de saúde ainda mantêm o enfoque na atenção curativa, individual, centrada na doença, com certo desconhecimento da realidade e do contexto sociocultural das famílias. Isto significa dizer que a atenção básica deveria trabalhar o conceito saúde nas diversas dimensões de sua abordagem bio-psico-social.

A dimensão da saúde mental está implicada nesta perspectiva ampliada da atenção à saúde. Nas intervenções demanda-se planejamento de ações e estratégias para a implementação, tanto quanto políticas de formação/capacitação de recursos humanos que possibilitem ao futuro profissional de saúde identificar e realizar ações nesta área, incluindo os diversos fatores de risco e situações que levam aos danos psíquicos e ao sofrimento do usuário do Sistema de Saúde, de forma que as demandas possam ser diagnosticados e tratados de forma preventiva e interventiva, principalmente na ESF.

Neste panorama, destaca-se a magnitude da abrangência societária do uso de álcool como instrumento de socialização, sendo relevante o monitoramento do uso do álcool(2). O contexto da formação do profissional de saúde, pautado no modelo polarizado entre saúde e doença, apresenta certa discrepância ao sobrevalorizar os quadros de dependência do álcool ou de outras drogas enquanto problema de saúde pública. A abordagem preconizada pela Organização Mundial de Saúde ${ }^{(2)}$ pauta-se em um continuum de quatro padrões de consumo, que podem ser visualizados pelos profissionais de saúde, quais sejam: uso de baixo risco, uso de risco, uso nocivo e dependência ${ }^{(2)}$.

Para cada padrão existem estratégias de intervenções à disposição, tanto preventivas como curativas. A atuação do profissional da saúde deve se pautar numa perspectiva fundamentada na confiança e na colaboração do próprio paciente.

Este enfoque parte de uma abordagem ampliada e distante do reducionismo do diagnóstico e das intervenções puramente medicamentosas. Ao levar em consideração o contexto de vida do sujeito e seu envolvimento no processo de intervenção, mostra-se como importante recurso a ser utilizado pelos enfermeiros, devendo estar presente tanto na formação como na agenda diária de trabalho, incorporando o tema dos problemas relacionados ao álcool nas práticas da ESF, uma necessidade à sua superação ${ }^{(3)}$.

Diante da importância desse contexto, revisou-se a literatura específica sobre dependência química na temática do uso de álcool, bem como nos manuais da ESF, sendo identificada lacuna sobre esse aspecto da saúde ampliada na APS, ênfase para o desenvolvimento do presente estudo.

Neste sentido, o presente artigo pretende identificar a concepção dos enfermeiros da ESF sobre a temática do uso problemático de álcool, em um contexto amazônico. Também, como esses profissionais trabalham o tema no cotidiano, no que se refere à população que faz uso desta substância psicoativa.

\section{MÉTODO}

Trata-se de um estudo de abordagem qualitativa, buscando a compreensão de determinado problema, na perspectiva daqueles que o vivenciam, assim como a natureza essencial dos fatos ${ }^{(4)}$. Os estudos qualitativos têm a capacidade de incorporar significado e intenção aos atos, às relações e às estruturas sociais, tanto no advento como na sua transformação, por imergirem nas construções humanas significativas ${ }^{(4)}$.

Para levantar as informações empíricas, foram utilizadas duas técnicas de coleta - Grupo Focal e Entrevistas Individuais semi-estruturadas ${ }^{(4-5)}$. O uso simultâneo destas técnicas permite levantar diferentes perspectivas da mesma temática, sendo que a primeira técnica refere-se a uma construção coletiva, enquanto a segunda à perspectiva individual dos entrevistados. O uso conjunto de ambas possibilita a confirmação ou não das impressões, com aprofundamento na interpretação das informações ${ }^{(5)}$. Os resultados foram discutidos de maneira integrada, na busca de uma perspectiva ampliada sobre o fenômeno investigado ${ }^{(5)}$.

O corpo de sujeitos da pesquisa constituiu-se dos doze enfermeiros da ESF no município de Coari - Região do Médio Rio Solimões (Estado do Amazonas), representando a totalidade destes profissionais no âmbito da APS. Para uma das técnicas empregadas, os Roteiros semi estruturados utilizados referiram-se aos diversos aspectos de como a temática do uso do álcool emerge no trabalho diário 
na ESF; o lidar com esta temática; e sobre a existência de ações individuais e da equipe no manejo do uso da substância pela população adscrita.

As seguintes características foram utilizadas critérios de inclusão: (1) ser enfermeiro; (2) trabalhar na ESF. A inadequação a qualquer um dos critérios acima excluiria o participante da pesquisa. Os entrevistados foram recrutados individualmente, através do contato dos pesquisadores na equipe em que atuavam. Todos convidados aceitaram participar do estudo.

Os Grupos Focais foram realizados em datas previamente agendadas, em sala cedida pelo Instituto de Saúde e Biotecnologia da Universidade Federal do Amazonas, no município de Coari (AM). Foram realizados dois grupos focais, cada um com seis sujeitos, com média de uma hora de duração, registrados com o uso de mini-gravadores digital. Os grupos foram conduzidos por um moderador, e acompanhados por um observador que anotava suas impressões e as expressões dos participantes. Durante o grupo, ambos sumarizavam os apontamentos dos participantes. Ao término, ambos os pesquisadores confeccionaram relatório de consenso, para auxílio na análise dos dados, conforme procedimentos estabelecidos pela literatura( ${ }^{(5)}$. Previamente a realização dos Grupos Focais, tanto o moderador como o observador passaram por capacitação na temática e por treinamento em condução de grupos focais. Decorridas duas semanas da realização dos Grupos Focais, os enfermeiros foram novamente contatados e agendaram-se as Entrevistas Individuais, realizadas com todos os participantes. Nesta oportunidade, obteve-se aprofundamento e complementação das informações coletadas durante o emprego da primeira técnica.

Como forma de identificação das falas selecionadas para o presente artigo, atribui-se o código GF para as oriundas dos Grupos Focais e El para aquelas das Entrevistas Individuais. A numeração, que se segue ao código, indica especificamente a que Grupo Focal ou Entrevista Individual pertence à fala, entre o material empírico total produzido durante a pesquisa.

O projeto foi submetido ao Comitê de Ética em Pesquisa com Seres Humanos da Universidade Federal do Amazonas, obtendo protocolo de aprovação ética, registrado sob o número CEP/UFAM/Parecer 114/2007. Todos os sujeitos participaram após explicação, compreensão e registro de aceitação formal em integrar a pesquisa pelo termo de consentimento livre e esclarecido, em duas vias (uma ao pesquisador e outra mantendo consigo). Foram seguidos todos os procedimentos éticos previstos na legislação vigente no país.

\section{RESULTADOS}

Os enfermeiros referem o uso de álcool como uma doença, focando no tratamento. Para os entrevistados, os indivíduos acometidos não têm domínio sobre suas ações e negam que estão doentes. Ao adorarem esta perspectiva, primam pela abordagem do uso do álcool como doença, não identificando os padrões de uso/risco contínuos. Apesar da referência ao fenômeno na dimensão mental da saúde e suas implicações, a conceituação destes profissionais fundamenta-se no modelo biomédico, existindo até a representação de contaminação pela morbidade.

É um problema de saúde pública. Alcoolismo é considerado uma doença (GF2).

Eu vejo o álcool como uma doença que precisa ser tratada com pessoas que já estão cronicamente contaminados (GF2).

Na verdade, todo mundo sabe que é uma doença. Quem conhece, entendeu que, na verdade, precisa mesmo é de ajuda (GF2)

O paciente não aceita que é doença (GF1).

O consumo excessivo de álcool foi apresentado como principal causa dos problemas relacionados à substância, relacionado à falta de controle do indivíduo sobre seu próprio modo de beber. $\mathrm{O}$ gradativo aumento da tolerância ao álcool é um emblema de que o sujeito é alcoolista para os entrevistados, desconsiderando o continuum existente entre o uso de baixo risco à dependência. Entre os entrevistados, a dosagem em si é tomada como parâmetro de dependência. Este conceito encontra-se em desacordo inclusive com os parâmetros clínicos em Psiquiatria sobre a adicção de álcool, segundo os quais, para a caracterização de dependência, é necessária a existência associada de três ou mais dos seguintes sintomas: forte desejo ou compulsão de beber; dificuldade de controle do uso; aumento de tolerância; interesses alternativos em favor do álcool. Há certa polarização entre estar sóbrio e ser alcoolista, refletindo o silencia sobre outras perspectivas e formas de manejo relacionadas ao álcool.

\begin{abstract}
No momento que eles têm não sabem dosar o tempo e a quantidade que vão ingerir daquele álcool. Eu acho que no momento que a pessoa bebe, já não é mais por numa festa ou socialmente. Ele bebe porque necessita, porque o organismo dele tipo cobra mais. Aí ele já é considerado um alcoólatra (EI5)
\end{abstract}

Quando é consumido em excesso, porque a partir do momento que a pessoa toma um copo, ela ainda não é considerada alcoólatra. Mas a partir do momento que passa de um copo já esta, pelo teste do bafômetro, alcoolizado (El8).

As implicações do uso de álcool não são restritas ao indivíduo em si. Afetam suas relações sociais, o âmbito familiar e a sociedade, sejam pelas implicações financeiras ou pela natureza emocional. Para os entrevistados, estas conseqüências justificam a abordagem da temática como problema de saúde pública, tanto em termos do sofrimento do indivíduo exposto, como pelo impacto familiar e societário. No entanto, denunciam falta de suporte para atuar frente a esta realidade, incluindo certo sentimento de culpa.

[...] com certeza vai repercutir em outro problema pra sociedade, que acaba sendo uma responsabilidade também de saúde pública. [...] geralmente nós temos que envolver
Enfermeiro de Saúde da Família na Amazônia: conceitos e manejo na temática do uso de álcool Moretti-Pires RO, Ferro SBG, Büchele F, Oliveira HM, Gonçalves MJF 
a família, o núcleo familiar, que aí nós vamos chegar à causa do problema para resolver (EI2).

$\mathrm{Na}$ realidade, é um problema. Como atinge ao todo a questão social, a questão familiar não deixa de estar inserida na sociedade. Falando especificamente da problemática patológica, a questão familiar é atingida, a questão cultural também e na realidade a comunidade teria que ter um programa específico para essa problemática. Infelizmente por culpa nossa ou falta de oportunidade, não foi inserida pra esse atendimento (EI7).

Entre os profissionais entrevistados, há claro desconhecimento sobre os dados nacionais da prevalência de uso e problemas relacionados ao álcool. É interessante notar a falta de referência/contra-referência da APS, havendo apenas a menção à Associação dos Alcoólicos Anônimos como iniciativa potencial de apoio aos casos no município. Estas informações diferem sobremaneira da perspectiva de que a APS, no âmbito individual e coletivo, se constitui como espaço para ações que abranjam promoção e proteção, prevenção de agravos, diagnóstico, tratamento, reabilitação e manutenção da saúde, desenvolvidas por práticas gerenciais e sanitárias democráticas e participativas, pautadas tanto no conhecimento do perfil epidemiológico da população assistida como nos princípios norteadores de referência/contra-referência e intersetorialidade.

Bem, o alcoólatra em si é uma pessoa que não vê o álcool. Se tu fores pegar estatísticas, não tem estatística sobre o álcool no Brasil, e no nosso município também provavelmente não. O certo que deve ter, que eu não conheço, são os alcoólicos anônimos em Coari (El1).

Existe os alcoólicos anônimos, mas o SUS, pelo menos até onde eu sei, não. Se existe eu desconheço trabalho específico, diretamente voltado para o álcool. Ás vezes as ONG's têm o tom mais iniciativo relacionado a isso (E/4).

A hereditariedade foi apontada como fator relacionado ao uso problemático de álcool. Porém, a falta de diálogo e de contato entre pai e filhos também mediariam o problema. Outra contribuição familiar se refere à reprodução de padrões de comportamento nas gerações, em que os filhos de bebedores também se comportariam dessa forma. 0 uso do álcool também apresenta a finalidade de sociabilização na qual, através da substância, os usuários facilitariam a interação com outros, em ambientes relacionais.

Às vezes, o jovem chega numa certa idade que não ouve pai e mãe infelizmente. Porque ele já tem um coleguinha da rua fazendo e acha que tem que fazer também (GF1).

Passa de pai para filho, porque o filho cresce com o pai bêbado dentro de casa, fica olhando, e às vezes até procura. É um refúgio (GF2).

Porque ele vê o pai, ele vê o tio, vê alguém da família fazendo ou até porque acha bonito mesmo (GF2).

Na escola não dá para dar educação de berço e sim orientação profissional. Educação familiar que é responsável. Então, quem usa o álcool, a culpa é dos pais porque eles não têm um alicerce familiar (GF1).

No seio da questão, os enfermeiros identificam a mídia como uma forte influência no padrão de uso de álcool da população. Apresentam as propagandas como portadoras da mensagem do bem estar imediato e momentâneo trazido pelo uso da substância, ao pautarem-se exclusivamente na descontração, diversão, mostrando pessoas bonitas e felizes. No entanto, as propagandas omitem/ ignoram as conseqüências diretas ocasionadas pelo uso álcool, tanto quanto as comorbidades associadas.

E a mídia mostra muitas mulheres bonitas, mulheres perfeitas. É o logotipo, é a mulher. [...] a própria mídia faz questão de fazer uma propaganda bonita, colocando artistas e influenciando a juventude a beber (GF1).

A mídia não quer perder. Para mídia não interessa que a aquela família não tem dinheiro para comer, que a família esteja pagando esse preço em relação ao álcool. O que a mídia está querendo, o que o produtor da TV, do vinho, da pinga querem fazer é vender, é ganhar dinheiro com isso. Daí as conseqüências, eles estão nem aí (GF2).

O Quadro 1 apresenta a síntese das informações empíricas, identificando convergências entre o produto coletivo dos Grupos Focais e as perspectivas das Entrevistas Individuais.

Quadro 1 - Síntese das categorias que emergiram dos grupos focais e das entrevistas individuais, identificando a convergência dos achados entre a perspectiva coletiva e a individual

\begin{tabular}{|c|c|c|}
\hline $\begin{array}{l}\text { Produtos } \\
\text { do Grupo Focal }\end{array}$ & $\begin{array}{c}\text { Informações nas } \\
\text { entrevistas semi-estruturadas }\end{array}$ & Convergência \\
\hline $\begin{array}{l}\text { - Ingestão em excesso como principal causa da } \\
\text { adicção por álcool } \\
\text { - Mídia influencia no uso de álcool pela } \\
\text { comunidade } \\
\text { - A mídia deveria fazer programas educativos para } \\
\text { a temática } \\
\text { - Necessidade de palestras e intervenções } \\
\text { - Apenas apontam a dependência e não os demais } \\
\text { padrões } \\
\text { - Sentimento de culpabilidade dos enfermeiros de } \\
\text { saúde da família } \\
\text { - Vitimização dos usuários dos serviços } \\
\text { - Uso de álcool é genético } \\
\text { - Álcool como doença } \\
\text { - Indivíduos não aceitam que estão doentes }\end{array}$ & $\begin{array}{l}\text { - Problema relacionado ao uso do álcool é } \\
\text { ocasionado pela ingestão em excesso } \\
\text { - Necessidade de conscientização do SUS sobre } \\
\text { o problema } \\
\text { - Não existem estatísticas sobre o uso de álcool } \\
\text { na Atenção Primária } \\
\text { - Necessidade de programas específicos } \\
\text { - Indicam apenas os alcoólicos anônimos como } \\
\text { outra instância } \\
\text { - Uso de álcool interfere na vida familiar } \\
\text { - Necessidade de sensibilizar a sociedade sobre } \\
\text { os danos causados pelo álcool }\end{array}$ & $\begin{array}{l}\text { Os enfermeiros não demonstraram preparação para } \\
\text { atuar frente ao uso problemático de álcool no âmbito } \\
\text { da atenção primária, mesmo sendo uma temática } \\
\text { abrangente em muitos aspectos. É extremamente } \\
\text { importante a participação diária do enfermeiro, a fim } \\
\text { de promover mudanças dos padrões de uso dos } \\
\text { usuários do SUS. A problemática do álcool é a } \\
\text { ingestão em excesso. A falta de intervenções na } \\
\text { problemática é marcante no discurso dos entrevista- } \\
\text { dos. O conhecimento do enfermeiro se baseia no } \\
\text { padrão de provável dependência e não dos demais que } \\
\text { antecedem a esta. Estes enfermeiros apresentam como } \\
\text { referência apenas a existência da Associação dos } \\
\text { alcoólicos anônimos no município. }\end{array}$ \\
\hline
\end{tabular}


As informações levantadas apontam para a questão da formação e educação permanente dos enfermeiros que atuam na ESF, no contexto amazônico investigado.

\section{DISCUSSÃO}

A conceituação dos entrevistados sobre o uso de Álcool como doença, é uma perspectiva que, de certa forma, reivindica a legitimidade do poder social dos profissionais de saúde, como se a temática se referisse ao adoecer e ao curar $^{(6)}$, e não aos laços profundos entre os diversos aspectos da socialização, a diferença significativa entre o bebedor de bar e o bebedor solitário, ou mesmo a questão do uso de álcool como emblema de status, controle de comportamento e das minúcias do poder ${ }^{(6)}$.

Paralelamente, a abordagem dos problemas de saúde pelos aspectos estritamente biológicos no uso do álcool e sua relação com outras morbidades, revela a manutenção de uma formação tecnicista e hospitalocêntrica, distante da formação integral e generalista ${ }^{(7)}$. É um indício da falta de preparo para lidar com dimensões amplas de saúde, como os padrões de uso anteriores à dependência e suas diversas implicações. Em outra perspectiva, a formação tradicional dos profissionais de saúde prioriza os protocolos biologicistas e o encaminhamento aos especialistas na APS ${ }^{(8)}$.

Um achado interessante é a estigmatização do usuário do álcool. Pode originar-se da dificuldade para adequar a transversalidade do tema ao paradigma da explicação dos motivos que levam o sujeito a adicção, isolando, de maneira errônea, o alcoolismo como fenômeno puramente social e desvinculado de outras temáticas como família, emprego, saúde geral, cultura.

A utilização de termos estigmatizados socialmente como alcoólatra; a referência ao fenômeno como transmissível de certa maneira - haja vista à menção da contaminação; e outros aspectos apresentados são muito semelhantes às representações sociais do alcoolista para familiares e para a sociedade. É um quadro díspar com a postura de profissionais que apresentam visão ampliada do processo saúde-doença, que é manifestação social, além de construção individual. Assim como os resultados do presente estudo, indícios na literatura apontam que a abordagem do enfermeiro na temática do uso de álcool ainda está focada nos sintomas e não na identificação precoce e prevenção de danos relacionados ${ }^{(9)}$.

Escapa-lhes que o uso de substâncias que alteram a consciência relaciona-se também a dimensão simbólica, ao conjunto das motivações do consumidor, que é sujeito ativo ao utilizar símbolos para se relacionar e se comunicar com seu meio social|(10). O álcool não pode ser tomado como uma substância de comercialização como outra qualquer. Historicamente, adquiriu importância oportunizada pelos aspectos culturais e simbólicos, na maioria das populações ${ }^{(11)}$. Encarar o uso apenas pela perspectiva da patologia é reduzir o tema e, por conseqüência desta simplificação, diminuir as possibilidades de intervenção junto à temática ${ }^{(10)}$.
Uma demanda importante identificada diz respeito aos mecanismos de referência/contra-referência para os casos. Uma vez que o SUS dispõe dos Centros de Atenção Psico-Sociais (CAPS), inclusive com modalidade específica para Álcool e Drogas - os CAPSs-AD, a inexistência destes no Médio Solimões constitui-se como uma importante barreira para os profissionais na ESF. São serviços de referência fundamentais para a atuação na temática e que deveriam estar à disposição de todas as Equipes de Saúde da Família ${ }^{(12)}$.

Estas reflexões encaminham para a idealização das ações na integralidade, vez que o SUS se propõe a atender às necessidades reais da população brasileira ${ }^{(1)}$. Em especial, os enfermeiros de Saúde da Família encontram problemas para uma prática em consonância com os já evocados princípios do SUS. Se tomado como emblema o uso do álcool pela população adscrita, como uma das dimensões da atenção à saúde de maneira integral, há problemas com a ação da ESF no Médio Solimões, tendo em vista que o álcool está relacionado à etiologia de $1,5 \%$ das mortes em termos mundiais ${ }^{(2)}$ e a prevalência de dependência de álcool é estimada em $11,2 \%$ dos adultos no Brasil ${ }^{(13)}$.

Ainda em termos de causalidade e uso de álcool, a hereditariedade associada ao uso de álcool é um fenômeno multifatorial/multicausal, havendo variações genéticas na aptidão ou resistência do organismo para sua manifestação ${ }^{(14)}$. No entanto, existem interações genéticas, sociais, culturais e ambientais que fundamentam seu desenvolvimento ${ }^{(14)}$, havendo indícios de que o bom relacionamento familiar e a prática religiosa são fatores de proteção para o desenvolvimento do uso problemático de álcool ${ }^{(15)}$.

Apesar da exígua literatura brasileira a respeito, estudos epidemiológicos longitudinais, em diversos países e contextos culturais, apontam que há associação entre a exposição às comunicações comerciais sobre álcool veiculadas pela Mídia e a inicialização/aumento do consumo da substância ${ }^{(16)}$, corroborando com a perspectiva levantada pelos entrevistados no presente artigo.

A despeito dos problemas de formação, os enfermeiros entrevistados mostraram-se sensibilizados sobre o assunto álcool. Referem sobre as dificuldades para ações de atenção neste campo. Há, portanto, predisposição destes profissionais para implantar intervenções na ESF. A atuação multiprofissional/interdisciplinar torna o modelo de atenção propício para agir frente à problemática. No entanto, além da capacitação, o processo de trabalho em Saúde da Família deve ser revisado e redimensionado, possibilitando não apenas a ação, mas também o acompanhamento e controle, a exemplo da inadequação e não confiabilidade dos dados dos Sistemas de Informações em Saúde atuais, no que se relaciona ao uso de álcool e outras substâncias ${ }^{(8)}$.

A atuação do profissional da ESF, coerente com a dinâmica desta problemática, deve pautar-se na intervenção apropriada e personalizada, fundamentando-se nos recur- 
sos de conscientização e motivação sobre o problema a partir do próprio paciente, cuja intervenção contemple as expectativas, os interesses e os valores de cada usuário, observada sua individualidade ${ }^{(6-7)}$.

Outra questão é a desconsideração da dimensão coletiva como passível de ação profissional na problemática. Não apenas para a comunidade, mas também para a família em especial, existem conseqüências tais como problemas no trabalho e desemprego, maus tratos, violência doméstica, acidentes, entre outros ${ }^{(8)}$. Por outro lado, dado o caráter social do uso desta substância e dos princípios norteadores da ESF, a coletividade é um campo interessante para diagnóstico e intervenção privilegiada pelas equipes de Saúde da Família.

Em toda América Latina, há certa tradição na Enfermagem em relação ao cuidado e prevenção no uso de drogas psicoativas. A despeito deste contexto, há que se caminhar em direção ao redimensionar dos conteúdos na graduação em Enfermagem com relação à temática aqui apresentada, principalmente no que se refira à Atenção Primária.

Autores $^{(17)}$ apresentam que $93 \%$ dos estudantes de Enfermagem, de uma amostra nacional de 25 escolas, receberam algum tipo de ensino sobre a problemática do álcool e drogas durante o curso. No que concerne ao conteúdo propriamente dito, $81 \%$ correspondeu ao enfoque psiquiátrico, $79 \%$ aos problemas relacionados à pessoa adulta e $72 \%$ ao fenômeno como doença. Em relação à conceituação sobre Dependência Química o estudo revela que apenas 1\% dos 485 entrevistados a relaciona aos problemas culturais, sociais e emocionais, sendo que o restante a atrela ao caráter biológico da dependência. Os autores sugerem a reformulação curricular, dada a falta de inclusão pertinente do tema nos cursos de Graduação em Enfermagem. As sugestões se referem tanto a transversalidade da temática, como a necessidade de um enfoque que priorize o trabalho contínuo e em equipe multiprofissional de natureza institucional e extra-institucional, apontando a necessidade de enfoque preventivo além do tratamento.

A formação universitária ainda é frágil na abordagem da problemática, pois $70 \%$ dos acadêmicos entrevistados receberam pouca ou nenhuma informação sobre problemas orgânicos, familiares e sociais relacionados ao álcool(18).

Cabe ressaltar que, a partir da década de 1990, o conhecimento científico para a temática em Enfermagem teve avanços indiscutíveis, inclusive no aspecto da formação dos enfermeiros ${ }^{(19)}$. Mas a formação e assistência, conforme descritas no presente trabalho, não acompanharam este movimento.

O panorama internacional sobre a formação do enfermeiro para lidar com problemas relacionados ao uso de álcool revela que as dificuldades não se restringem ao Brasil. Estudo realizado em Colômbia apresenta que, apesar do conhecimento teórico legado pela graduação e da tradição de cuidado que detém a Enfermagem, existem dificuldades para a atuação na prática cotidiana deste aporte, assim como do trabalho multiprofissional e integrado exigido para intervenções em uma perspectiva ampliada em saúde ${ }^{(20)}$.

Esta lacuna na atenção primária é passível de solução, existindo Estratégias preconizadas pela OMS no rastreamento e intervenção nesta problemática em Atenção Primária. São as chamadas Estratégias de Rastreamento e Intervenções Breves para uso problemático de álcool ${ }^{(7)}$. A utilização de intervenções breves tem por objetivo identificar usuários nos padrões contínuos e variados de uso do álcool, a fim de promover prevenção antes do desenvolvimento da síndrome da dependência, reduzindo o risco dos danos relacionados ao uso da substância psicoativa ${ }^{(7)}$.

A utilização de intervenções breves pauta-se na ação de aconselhamento, utilizando-se da empatia junto ao usuário que delas necessitem. Promovem a redução gradativa dos riscos de danos de maneira centrada no usuário, e não em protocolos estritamente biológicos. Com isso, o enfermeiro de Saúde da Família tem a sua disposição uma rotina de atendimento, tanto nas unidades de saúde assim como na comunidade, com abordagem que se pauta na identificação de padrões e prescrição de condutas, mas também no estímulo, sensibilização e motivação do usuário para sua própria redução de uso gradativa.

Dada a importância levantada desta temática, o presente trabalho se constitui como uma aproximação inicial, não havendo a presunção de esgotar todos os aspectos, inclusive por referir-se ao binômio formação/atuação dos enfermeiros de Saúde da Família frente ao uso da substância pela população adscrita.

Escapa da abrangência da presente investigação como esta temática está articulada nas demais profissões implicados na ESF, de sua formação/atuação, além dos outros setores envolvidos tais como o comércio local, o poder público e sua postura, o judiciário, os setores de repressão ao uso, entre outros que se referem ao objeto de investigação, que pode ser tomado como emblema de intersetorialidade em um tema de saúde tão presente na civilização ocidental.

\section{CONCLUSÃO}

A proposta de se criar um modelo de atenção como a Saúde da Família fundamenta-se em diversos princípios e, entre estes, um dos mais importantes: a ação da equipe junto aos principais problemas de saúde da população.

A problemática do uso de álcool na população adscrita à ESF não é apenas pertinente pelo perfil epidemiológico da população brasileira, mas pelas conseqüências a médio e longo prazo na saúde dos indivíduos, o impacto familiar e as decorrências para a sociedade, tais como violência, entre outras. Este panorama é apresentado pelos próprios entrevistados, apontando que os enfermeiros de Saúde da Família visualizam a importância e relevância da temática. 
No entanto, incoerências na implantação da ESF são apresentadas, principalmente no que se refere à falta de formação e educação permanente, denunciadas pelas entrevistadas e que comprometem seriamente as ações em atenção primária no SUS. No presente artigo este panorama apresenta-se no recorte do uso de álcool, mas não se restringe a este. Há premente necessidade de mudança nos currículos, na política, e na prática profissional, apon-

\section{REFERÊNCIAS}

1. Brasil. Ministério da Saúde. Secretaria de Gestão do Trabalho e de Educação na Saúde. Departamento de Educação na Saúde. Política de Educação e Desenvolvimento para o SUS: caminhos para a educação permanente em saúde. Brasília; 2004.

2. Babor TF, Higgins-Biddle JC, Saunders JB, Monteiro MG. AUDIT: teste para identificação de problemas relacionados ao uso de álcool: roteiro para uso em atenção primária. Ribeirão Preto: PAI-PAD; 2003.

3. Carmelo SHH. Riscos psicossociais relacionados ao estresse no trabalho das Equipes de Saúde da Família e estratégias de gerenciamento [tese doutorado]. Ribeirão Preto: Escola de Enfermagem de Ribeirão Preto, Universidade de São Paulo; 2006.

4. Minayo MCS. O desafio do conhecimento: pesquisa qualitativa em saúde. 6a ed. São Paulo: Hucitec; 1999.

5. Morgan D. Focus group: as qualitative research. 2nd ed. London: Sage; 1997.

6. Bucher R. Drogas e sociedade nos tempos de AIDS. Brasília: Ed. UnB; 1996.

7. Laranjeira R, Romano M. Consenso Brasileiro sobre Políticas Públicas do Álcool. Rev Bras Psiquiatr. 2004;26 Supl 1:68-77.

8. Nunes M, Jucá VJ, Valentim CPB. Ações de saúde mental no Programa Saúde da Família: confluências e dissonâncias das práticas com os princípios das reformas psiquiátrica e sanitária. Cad Saúde Pública. 2007;23(10):2375-84.

9. Carlini EA, Galduroz JCF, Noto AR, Nappo SA. I levantamento domiciliar sobre o uso de drogas psicotrópicas no Brasil: estudo envolvendo as 107 maiores cidades do país - 2001. São Paulo: CEBRID/UNIFESP; 2002.

10. Baú CHD. Estado atual e perspectivas da genética e epidemiologia do alcoolismo. Ciênc Saúde Coletiva. 2002;7(1):183-90.

11. Marlatt BC. Uso de drogas psicotrópicas no Brasil. In: Secretaria Nacional Antidrogas. Formação de multiplicadores de informações preventivas sobre drogas. Florianópolis: UFSC/ SENAD; 2002. p. 65-83. tando para o emprego prático de tecnologias leves no cotidiano do profissional da ESF.

A falta de suporte para os profissionais na temática, em termos de referência/contra-referência, também é apresentada como um nó crítico do contexto, tanto quanto a ausência de programas e serviços no âmbito da Estratégia Saúde da Família.

12. Anderson P, de Bruijn A, Angus K, Gordon R, Hastings G. Impact of alcohol adverting and media exposure on adolescent alcohol use: a systematic review of longitudinal studies. Alcohol Alcoholism. 2009;44(3):229-43.

13. Souza MLP. Expansão do PSF e identificação dos problemas relacionados ao abuso de álcool no Brasil. Rev Bras Psiquiatr. 2005;27(4):342-3.

14. Marques ACPR, Furtado EF. Intervenções breves para problemas relacionados ao álcool. Rev Bras Psiquiatr. 2004;26 Suppl 1:28-32.

15. Babor TF, Higgins-Biddle JC. Intervenções breves para uso de risco e risco nocivo de álcool: manual para uso em atenção primária. Ribeirão Preto: PAI-PAD; 2003.

16. Vargas D, Oliveira AF, Luís MAV. Atendimento ao alcoolista em serviços de atenção primária à saúde: percepções e condutas do enfermeiro. Acta Paul Enferm. 2010;23(1):73-9.

17. Ramos LH, Pillon SC, Cavalcante MBG, Luiz MV, Padredi FM, Laranjeira RR. O ensino sobre dependência química em cursos de graduação em enfermagem no Brasil, 1998. Acta Paul Enferm. 2001;14(3):35-43.

18. Pillon SC, Laranjeira RR. Formal education and nurses' attitudes towards alcohol and alcoholism in a Brazilian sample. Sao Paulo Med J. 2005;123(4):175-80.

19. Pinho PH, Oliveira MAF, Vargas D, Almeida MM, Machado AL, Aranha e Silva AL, et al. Reabilitação psicossocial dos usuários de álcool e outras drogas: a concepção de profissionais de saúde. Rev Esc Enferm USP. 2009;43(n.esp.2):1261-6.

20. Vásquez EM, Pillon SC. La formación de enfermeras y el fenomeno de las drogas en Colombia: conocimientos, actitudes y creencias. Rev Latino Am Enferm. 2005; 13(n.esp):845-53. 